\title{
Polarization Correlometry of Birefringence Images of Human Blood Layers
}

\author{
A.G. Ushenko ${ }^{1}$, P.O. Angelsky ${ }^{1}$, A.O. Karachevtsev ${ }^{1}$, G.B. Bodnar ${ }^{2}$, \\ G.D. Koval ${ }^{2}$, O.G. Prydiy ${ }^{1}$, Yu. Marchuk ${ }^{2}$ \\ ${ }^{1}$ Optics and Publishing Department, Chernivtsi National University, \\ 2 Kotsyubinsky Str., Chernivtsi, 58012, Ukraine \\ ${ }^{2}$ Bukovinian State Medical University, \\ alexander.dubolazov@gmail.com
}

\begin{abstract}
To analyze the coordinate-like structure of Stokes-parametric and Mueller-matrix images of optically anisotropic components of biological tissues, the two-point polarization-correlational approach was applied. On this basis parameters the method of cross-correlational definition of parameters (average sizes, asymmetry factor) of correlational contour was developed, which defines the topographical structure of the characteristic meanings of Stokes-parametric and Mueller-matrix images of histological sections of biological tissues.
\end{abstract}

Keywords: polarimetry, laser, biofluids, correlometry

1. Introduction. Traditionally the analysis of the processes of scattering electromagnetic radiation by optically inhomogeneous objects is studied with the help of two approaches - "scalar" (photometry and spectrophotometry) and "vector" (Mueller - matrix optics). Lee Hon Wang [1], Tuchin V. P. [2] and Angelskii O.V. [3] define the relationships between a set of statistical moments of the first and the fourth order that characterize phase-inhomogeneous layers and the division of their states of polarization of laser images. The works of Ushenko Yu. O. [4-6] demonstrate using such parameters when describing optical anisotropy of two-beam refractive chains of biological tissues on the basis of the correlation analysis of coordinate distributions of Mueller matrix elements - Mueller matrix images of histological sections of biological tissues of different morphological structures and physiological states. What is more, polarization correlometry needs further development. First of all, in order to expand the functionality of diagnosing optical anisotropy of biological layers, it's necessary to have both the synthesis of statistical (one-point) and correlation (two-point) approaches of analyzing topographical structure of Mueller`s matrix images and the fields of laser radiation, modified by the nets of biological crystals.

2. Correlation approach to describing the topographic structure of Mueller`s matrix images of polycrystalline networks

Using one-point singular approach to stokes-parametric images of polycrystalline networks, there will be a detailed analysis of coordinated distributions of extreme values $\beta(x, y)=0$ and $\beta(x, y)=\frac{\pi}{4}$ of ellipticity of the polarization of the corresponding images [7 - 19].

If viewing the analysis of coordinate distribution of homogeneously polarized object field in terms of two-point approach, it is necessary to consider the correlation interrelationships of all states of polarization within the image of our biological samples step by step. Such information is provided by the combined degree of mutual polarization (CDMP) of images of biological objects under research.

Within the frames of singularly-parametric approach to Mueller's matrix images of biological phaseinhomogeneous layers, another option, concerning the combined degree of mutual anisotropy (CDMA), which directly characterizes the correlation coherence of anisotropy parameters of different points of polycrystalline network. Characteristic values of CDMA are clearly associated with the relevant characteristic values of CDMP.

Eleventh International Conference on Correlation Optics, edited by Oleg V. Angelsky, Proc. of SPIE Vol. 9066, $906622 \cdot$ @ 2013 SPIE · CCC code: 0277-786X/13/\$18 · doi: 10.1117/12.2053928 
Table 1.Characteristic values of CDMA $\tilde{W}\left(r_{1}, r_{2}\right)$ of Mueller`s matrix images and CDMP $W\left(r_{1}, r_{2}\right)$ of stokes-parametric image of polycrystalline network.

\begin{tabular}{|c|c|c|c|}
\hline $\begin{array}{c}\gamma\left(r_{1}\right), \gamma\left(r_{2}\right) \\
(0 \div \pi)\end{array}$ & $\begin{array}{c}\varphi\left(r_{1}\right)=\varphi\left(r_{2}\right) \\
(0 \div \pi)\end{array}$ & $\tilde{W}\left(r_{1}, r_{2}\right)$ & $\widetilde{V}\left(r_{1}, r_{2}\right)$ \\
\hline \multirow{3}{*}{$\begin{array}{c}\gamma\left(r_{1}\right)=\gamma\left(r_{2}\right) \\
\gamma\left(r_{1}\right)=0,5 \pi+\gamma\left(r_{2}\right)\end{array}$} & 0 & 1,0 & 1,0 \\
\hline & $0,5 \pi$ & 1,0 & 1,0 \\
\hline & $\pi$ & 1,0 & 1,0 \\
\hline \multirow{4}{*}{$\begin{array}{c}\gamma\left(r_{1}\right)=\gamma\left(r_{2}\right), \\
\gamma\left(r_{2}\right)=0,5 \pi+\gamma\left(r_{2}\right)\end{array}$} & $\gamma\left(r_{2}\right)=0,5 \pi+\gamma\left(r_{2}\right)$ & $\widetilde{W}\left(r_{1}, r_{2}\right)$ & $\widetilde{V}\left(r_{1}, r_{2}\right)$ \\
\hline & $0,5 \pi$ & 0,5 & 0,5 \\
\hline & $\pi$ & 0,5 & 0,5 \\
\hline & $1,5 \pi$ & 0,5 & 0,5 \\
\hline \multirow{4}{*}{$\begin{array}{c}\gamma\left(r_{1}\right)=\gamma\left(r_{2}\right) \\
\gamma\left(r_{2}\right)=0,5 \pi+\gamma\left(r_{2}\right)\end{array}$} & $\gamma\left(r_{2}\right)=\pi+\gamma\left(r_{2}\right)$ & $\tilde{W}\left(r_{1}, r_{2}\right)$ & $\widetilde{V}\left(r_{1}, r_{2}\right)$ \\
\hline & $\pi$ & 0 & 0 \\
\hline & $1,5 \pi$ & 0 & 0 \\
\hline & 0 & 0 & 0 \\
\hline
\end{tabular}

\section{Method of measuring the correlation circuits of the networks of biological crystallites}

The method of determining the structure of the correlation contour is based on the cross-correlation analysis, which in turn is based on the definition of:

- two-dimensional autocorrelation function $J(x, y)$ of Mueller's matrix image $m_{i k}(x, y)$ with the further definition of its cross-section at the level of $J(\widetilde{W})=0,5$ that determines the locus of points of the correlation circuit;

- series of auto correlation division relationships between the elements of Mueller's matrix in two mutually orthogonal directions $-O x$ and $O y$ :

$$
\begin{gathered}
J(X, y) \Rightarrow\left(\begin{array}{l}
J_{1}(\Delta x) ; \Delta x=1, \ldots, m \\
J_{y=n}(\Delta x) ; \Delta y=1, \ldots, n
\end{array}\right) \Rightarrow \bar{J}(\Delta x)=\sum_{i=1}^{n} J_{i}(\Delta x) ; \\
J(x, Y) \Rightarrow\left(\begin{array}{l}
J_{1}(\Delta y) ; \Delta y=1, \ldots, n \\
J_{x=m}(\Delta y) ; \Delta y=1, \ldots, m
\end{array}\right) \Rightarrow \bar{J}(\Delta x)=\sum_{i=1}^{n} J_{i}(\Delta x) ;
\end{gathered}
$$

where $J_{i}(\Delta x), J_{j}(\Delta y)$ - autocorrelation functions calculated in mutually perpendicular directions of the plane of biological layer.

- Logarithmic dependencies of the spectra power distribution in terms of the values of Muller-matrix image of the birefringence of crystallite net in two mutually orthogonal directions $O x$ and $O y$

- On the basis of the asymmetry coefficient of the correlative loop of the Muller-matrix image of the biological sphere correlation (1) in two mutually perpendicular directions 


$$
\chi=\frac{L x}{L y}=\frac{J(\Delta x)=0,5}{J(\Delta y)=0,5}
$$

\section{Computer modeling of the formation of correlative loops of the birefringent nets}

Figure 1 provides the coordinate distributions of the complex level of mutual anisotropy (CLMA) of such objects and their two- dimensional autocorrelated function.

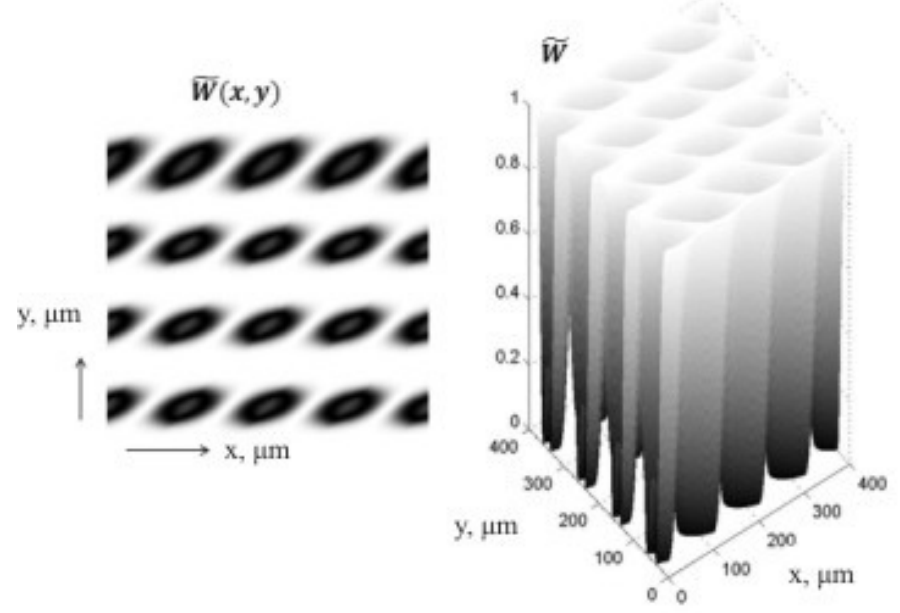

Figure 1. The distributions of CLMA net of the rectilinear cylinders with harmonious phase modulation.

The data obtained indicates the fact that even in a rather simple case of the deterministic orientation-phase modulation the complex coordinate distribution of CLMA is formed, which is characterized by the azimuthally asymmetrical autocorrelated function.

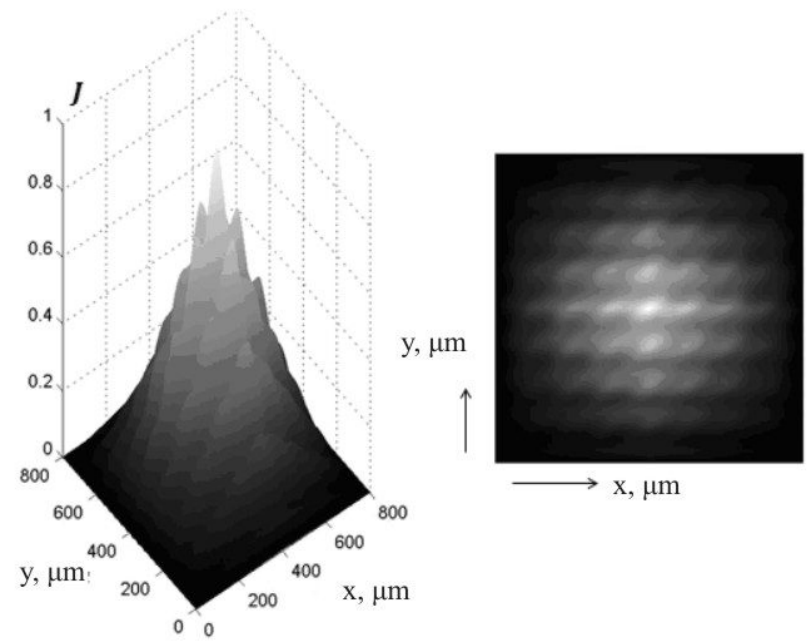

Figure. 2. Two-dimensional autocorrelated function of the coordinate distribution of the CLMA parameter of the rectilinear cylinder net with the harmonious phase modulation.

Figure three displays the configuration of the correlated loop of the rectilinear cylinders grid with the sinusoidal law of the phase modulation. 


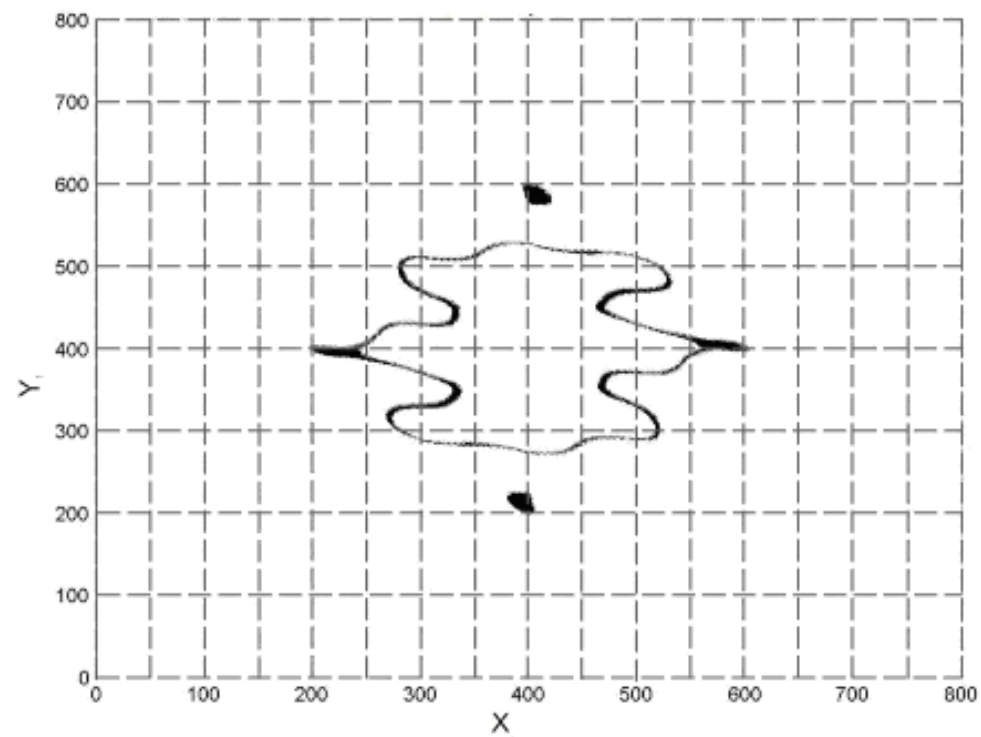

Figure. 3. Correlated loop of the coordinate distribution of the CLMA parameter of the rectilinear cylinders net with the harmonious phase modulation.

Apparently, the correlated loop of the rectilinear cylinders' net with the sinusoidal law of the modulation phase is a complex distribution of the geometrical place of the dots which correspond to the half-width of the two-dimensional autocorrelated function (figure.2) of the coordinate distribution of the pair-wise parameter that is a complex degree of mutual anisotropy (figure 3).

The utilization for the characteristic and differentiation of the birefringence nets of different types of the twodimensional coordinate dependencies of the correlated loop is to some extent extra difficult because of its azimuthally asymmetric construction.

That is why the configuration of such a loop continues to be estimated within the cross-correlated analysis in two orthogonal directions of the coordinate displacement of the two-dimensional distribution of the CLMA - figure 4 and 5 

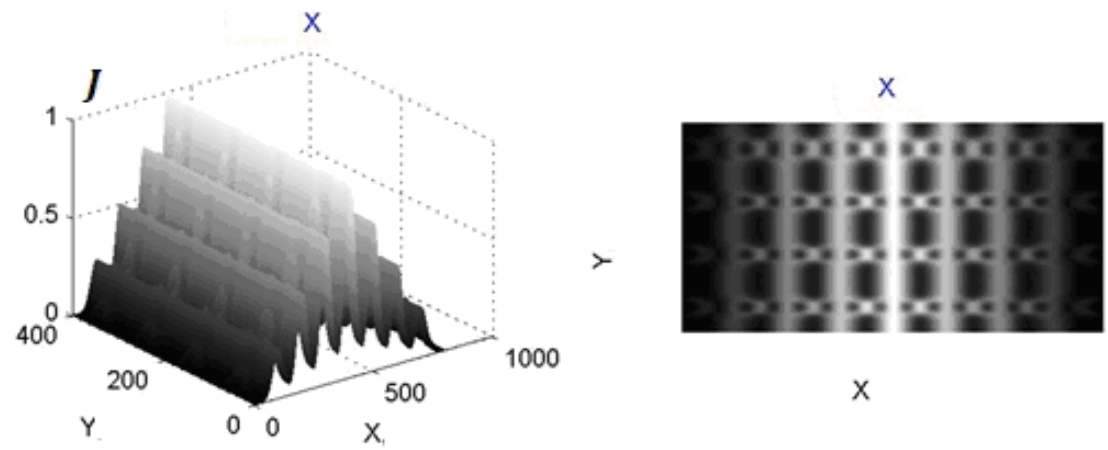

$\mathrm{X}$
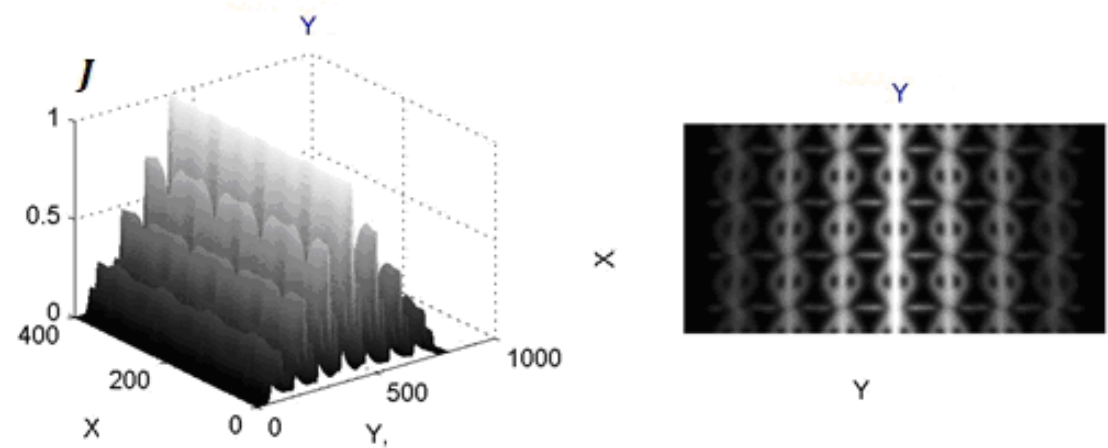

Figure 4. Cross-correlated functions of the coordinate distribution of the CLMA parameter of the rectilinear net cylinders with the harmonious phase modulation.

The two-dimensional cross-correlated functions that are displayed in figure 4 possess a complex and an individual CLMA parameter for each direction of scanning. The geometrical places of the dots that correspond to the half-width of the cross-correlated functions were defined in order to find out the average orthogonal dimensions of the correlated loop.

The results of such an approach are displayed in the figure 5 .
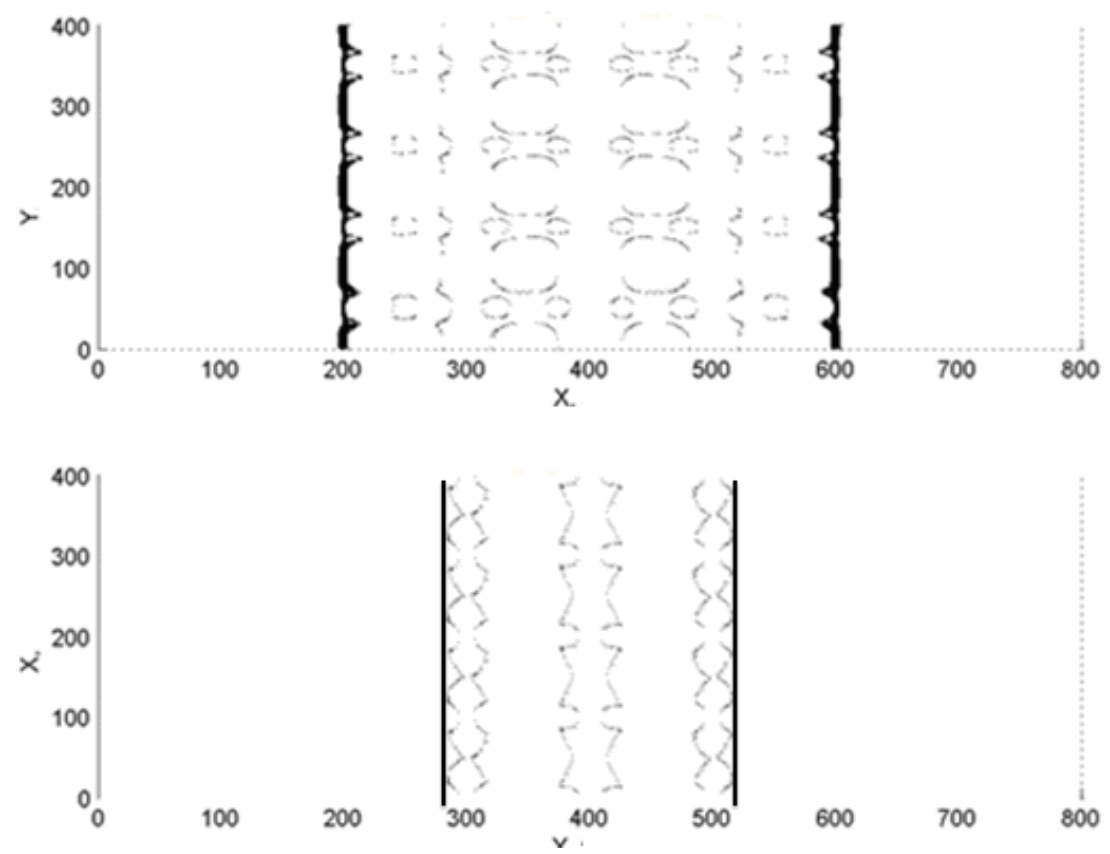

Figure 5. The orthogonal dimensions of the correlated loop of the coordinated distribution of the CLMA parameter that takes place in the rectilinear cylinders with the harmonious phase modulation. 
Evidently, the correlated loop of the coordinated CLMA parameter distribution of the net rectilinear cylinders with the sinusoidal phase modulation law possesses a significant asymmetry in two mutually perpendicular directions revealed in different average diametrical dimensions.

The elaborated method of the polarization-correlation analysis was approved in order to find out the benign and malignant changes of the biological tissues.

Figures 6 and 7 show the coordinate distributions of the laser images coefficient of the specific and relative absorption (CSRA) of the optically slim histological cuts of the operationally removed benign (fibromyoma - figure 6) and malignant (figure 7) tumor (adenocarcinoma) of the uterus.
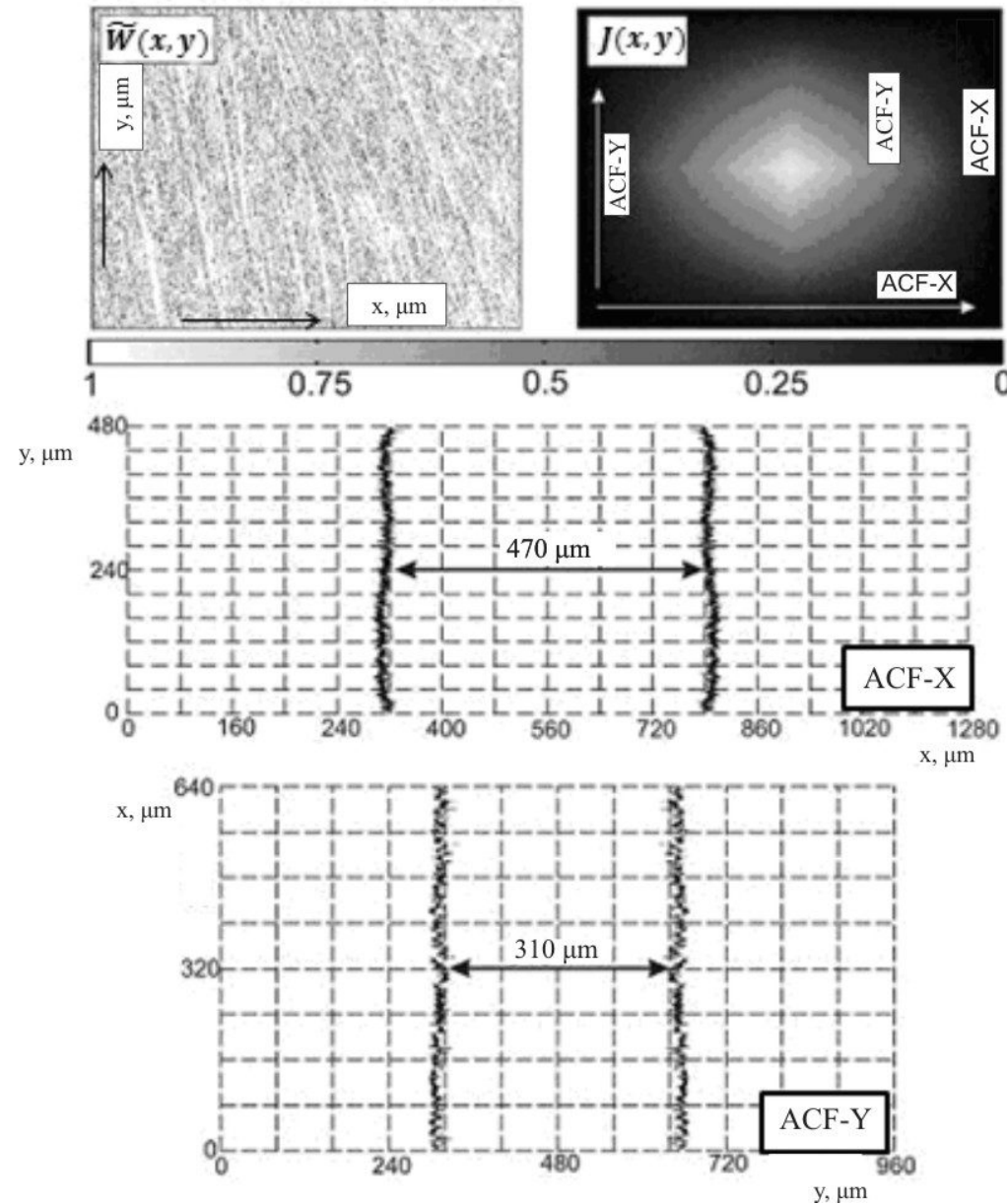

Figure. 6 Coordinate, autocorrelational and the cross-correlational structure of distribution of the CSRA laser images of the uterus wall tissue with the qualitative changes.

From the obtained data we can say that the ratio differs between the average sizes of the orthogonally measured in directions of correlation contours coordinate distributions CLMA of histological slices of good-quality and malignant changes of the uterus tissues.

A non-cancerous growth contour is asymmetrical due to the increase in of tworefrection fibrils.

In cancer the asymmetry of correlation contour is reduced by nearly double because of the destruction of tworefrection systems and corresponding azimuthal symmetrization of coordinate change in the state of polarisation of the laser image. 

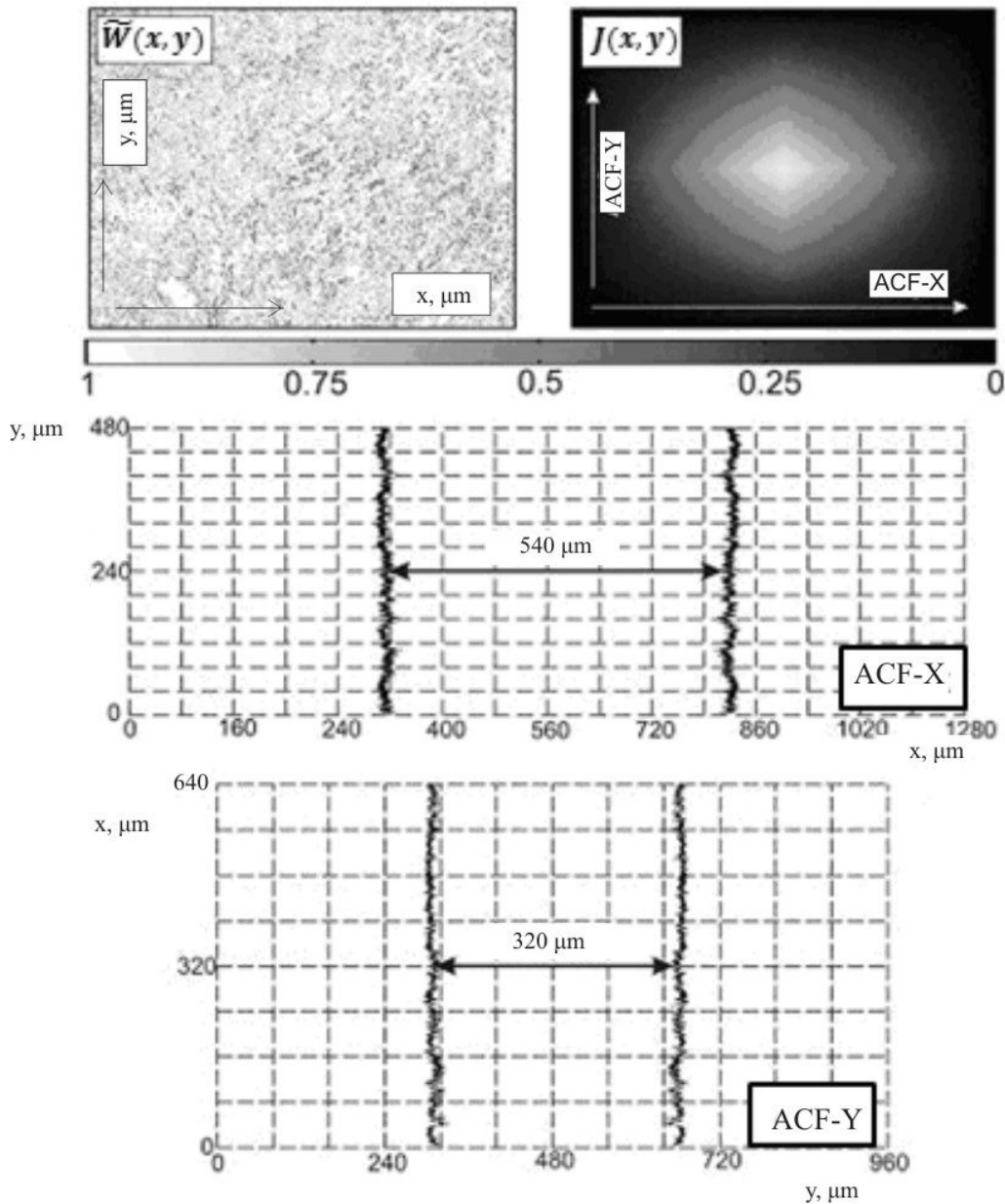

Figure. 7 Coordinate, autocorrelational and the cross-correlational structure of distribution of the CSRA of laser images of uterus wall tissue with malignant changes.

Quantitatively distinctiveness of cross-correlational structure of two-dimensional distributions of CSRA illustrates the excess of autocorrelational functions and the dispersion of logarithmic dependencies of power spectra are shown in table 2 .

Table 2. Parameters of cross-correlational analysis of CLMA distribution

\begin{tabular}{|c|c|c|}
\hline Parameters & Non-cancerous growth $(\mathrm{q}=11)$ & Cancerous growt $(\mathrm{q}=12)$ \\
\hline$\chi$ & $2,05 \pm 0,28$ & $0,93 \pm 0,014$ \\
\hline$K_{4}$ & $1,82 \pm 0,29$ & $0,37 \pm 0,046$ \\
\hline$\Omega$ & $0,23 \pm 0,037$ & $0,48 \pm 0,057$ \\
\hline
\end{tabular}

It is established that distinctiveness of correlational and spectral parameters defined for histological sections of the biopsy of tissue of both groups are 2 and 5 times, respectively, and this provides specificity of the cross - correlational method at the level of $80 \%$.

\section{Conclusions}

1. For the first time, to analyze the coordinate-like structure of Stokes-parametric and Mueller-matrix images of optically anisotropic components of biological tissues, the two-point polarization-correlational approach was 
applied. On this basis parameters the method of cross-correlational definition of parameters (average sizes, asymmetry factor) of correlational contour was developed, which defines the topographical structure of the characteristic meanings of Stokes-parametric and Mueller-matrix images of histological sections of biological tissues.

2. The relationship between one-point (polarization-singular) and two-point (polarization-correlational) approaches for the analysis of Stokes-parametric and Mueller-matrix images of biological polycrystal systems was found. It is established that the average transverse dimensions of the correlational contour of Stokes-parametric and Muellermatrix images are equal to the half-width of aggregate of orthogonal autocorrelational functions of coordinational distribution of a integrated degree of reciprocal anisotropy.

3. In the approximation of single scattering the relationship between quantitative parameters (the asymmetry coefficient of correlational contour of complex degree of mutual anisotropy, the correlational moment of 4-th degree and the spectral moment of the 2nd degree of distribution of a complex degree of mutual anisotropy) was found for the first time, which characterize the asymmetry of the correlational contour of Stokes-parametric and Mueller - matrix images and changes of tworefrection of biological tissues. On this basis for the first time the polarization-correlational differentiation was implemented for the first time on benign and malignant changes of a uterus tumor histological sections of the uterus.

\section{REFERENCES}

[1] Wang L.-H. Propagation of polarized light in birefringent turbid media: a Monte Carlo study / X. Wang, L. - H. Wang // J. Biomed. Opt. - 2002. - Vol. 7. - P. 279-290.

[2] Тучин В. В. Лазеры и волоконная техника в биомедицинских исследованиях / Тучин В. В. - Саратов: Изд-тво Сарат. ун-та, 1998. - 384c.

[3] Angelsky, O., V., Ushenko, A., G., Burcovets, D., N., Ushenko, Yu., A., "Polarization visualization and selection of biotissue image two-layer scattering medium," J. Biomed. Opt. 10(1), P. 014010 (2005).

[4] Ushenko, Yu.,O., Dubolazov, O., V., Karachevtsev, A.,O., Gorsky, M., P., Marchuk, Yu., F., "Wavelet analysis of Fourier polarized images of the human bile," Applied Optics (51), P. 133-139 (2012).

[5] Angelsky, O.,V., Hanson, S., G., Zenkova, C.,Yu., Gorsky, M.,P., Gorodyns'ka, N.,V., "On polarization metrology (estimation) of the degree of coherence of optical waves," Optics Express 17(18), pp.15623-15634 (2009).

[6] Angelsky, O.,V., Burkovets, D.,N., Kovalchuk, A.,V., Hanson, S.,G., "Fractal description of rough surfaces," Applied optics 41 (22), 4620-4629 (2002).

[7] Angel'skii, O.,V., Ushenko, O.,G., Burkovets, D.,N., Arkhelyuk, O.,D., Ushenko, Y.,A., "Polarization-correlation studies of multifractal structures in biotissues and diagnostics of their pathologic changes," LASER PHYSICS 10(5), 1136-1142 (2000).

[8] Angel'skii, O.,V., Ushenko, A.,G., Arkhelyuk, A.,D., Ermolenko, S.,B., Burkovets, D.,N., "Scattering of laser radiation by multifractal biological structures," Optika i Spektroskopiya 88 (3), 495-498 (2000).

[9] Angel'skii, O., V., A. G. Ushenko, A. D. Arkhelyuk, S. B. Ermolenko, D. N. Burkovets and Yu. A. Ushenko "Laser polarimetry of pathological changes in biotissues," Optika i Spectroskopiya 89( 6), 1050-1055 (2000).

[10] Angelsky, O.,V., Yermolenko, S.,B., Zenkova, C.,Yu., Angelskaya, A.,O., "Polarization manifestations of correlation (intrinsic coherence) of optical fields," Applied optics 47 (29), 5492-5499 (2008)

[11] Angelsky, O.,V., Polyanskii, P.,V., Felde, C.,V., "The emerging field of correlation optics,"Optics and Photonics News 23(4), p.p.25-29 (2012).

[12] Bekshaev, A., Y., Angelsky, O., V., Hanson, S., G., Zenkova, C., Y., "Scattering of inhomogeneous circularly polarized optical field and mechanical manifestation of the internal energy flows," Phys. Rev. A. (86), 023847 (2012).

[13] Arkhelyuk, A., Podkamen, L., "The influence of optical parameters of intermediate medium on the extinction matrix of oriented particles layer," SPIE Proc. 6254, p.293-298 (2006).

[14] Brus, V., Pidkamin, L.,J., Abashin, S.,L., Kovalyuk, Z.,D., Maryanchuk, P.,D., Chugai, O.,M., "Optical constants and polarimetric properties of TiO2-MnO2 thin films," Optical Materials (34), 1940-1945 (2012).

[15] Ushenko, Y.,A., Tomka, Y.,Y., Dubolazov, A.,V., "Laser diagnostics of anisotropy in birefringent networks of biological tissues in different physiological conditions," Quantum Electronics 41 (2), p. 170-175 (2011).

[16] Ushenko, Y.,A., Dubolazov, O.,V., Karachevtsev, A.,O., "Statistical structure of skin derma Mueller matrix images in the process of cancer changes," Optical Memory \& Neural Networks 20 (2), p.145-154 (2011).

[17] Angelsky, O., Mokhun, A., Mokhun, I., Soskin, M., "The relationship between topological characteristics of component vortices and polarization singularities," Optics communications 207 (1), 57-65 (2002). 
[18] Kushnerick, L., Ya., Bodnar, L., B., Gorskii, M., P., Sydor, M., "Statistical and Correlation Structure of Fourier Spectra Polarizationally Filtered Laser Images of Optically Anisotropic Biological Networks," Optical Memory and Neural Networks (Information Optics) 22(2), pp. 118-125 (2013).

[19] Koval, G.,D., Raranskiy, M.,D., "System of space-frequency filtering of linear and circular birefringence in cancer diagnosis," Proc. SPIE 8842 (Novel Optical Systems Design and Optimization XVI), 884212 (2013). 